\title{
OSCILLATION CRITERIA FOR NONLINEAR SECOND ORDER MATRIX DIFFERENTIAL EQUATIONS
}

\author{
GARRET J. ETGEN
}

ABSTRACr. The object of this paper is to present sufficient conditions for the oscillation of certain solutions of the second order, noniinear matrix differential equation. The oscillation criteria obtained here improve the recent results of the author and E. C. Tomastik. The methods employed in the paper extend a technique introduced by $\mathrm{H}$. C. Howard and for the special linear version of the nonlinear equation, the resulting oscillation criteria represent improvement of Howard's work. Using an extension of the unitary transformation introduced by F. V. Atkinson, estimates of the oscillation of solutions aie obtained.

Introduction. This paper is concerned with the following special system of $2 n^{2}$ nonlinear first order differential equations:

$$
\begin{aligned}
y_{i j}^{\prime} & =\sum_{h=1}^{n} k_{i h}\left(x, y_{11}, y_{12}, \cdots, y_{n n}, z_{11}, z_{12}, \cdots, z_{n n}\right) z_{h j}, \\
z_{i j}^{\prime} & =\sum_{h=1}^{n} g_{i h}\left(x, y_{11}, y_{12}, \cdots, y_{n n}, z_{11}, z_{12}, \cdots, z_{n n}\right) y_{h j},
\end{aligned}
$$

$1 \leqq i, j \leqq n, n>1$, defined on the interval $[a, \infty)$, where the functions $k_{i j}$ and $g_{i j}, i, j=1,2, \cdots, n$, are real-valued, continuous and satisfy conditions which will insure a unique solution when appropriate initial conditions are specified. In addition, it will be assumed that $k_{i j} \equiv k_{j i}$ and $g_{i j} \equiv g_{j i}$ for all $i, j$. This system shall be represented in the matrix form

$$
Y^{\prime}=K(x ; Y ; Z) Z, \quad Z^{\prime}=-G(x ; Y ; Z) Y .
$$

Special cases of (1) in which each of $K$ and $G$ is positive definite for all $x$ on $[a, \infty)$ and for all differentiable pairs of matrices $\{Y(x), Z(x)\}$, have been considered by E. C. Tomastik [6] and by the author [2], [3]. In these papers certain oscillatory properties of the solution pairs have been obtained.

The purpose of this paper is to extend the oscillation results contained in [2], [3], [6], as well as the results of H. C. Howard [5]. In particular, the condition that each of $K$ and $G$ be positive definite

Received by the editors October 13, 1969.

AMS 1969 subject classifications. Primary 3420, 3426, 3442.

Key words and phrases. Nonlinear matrix differential equations, second order matrix differential equations, oscillation criteria, estimates of oscillation. 
will be relaxed to the condition that at least one of $K$ and $G$ be positive definite. Based on the method introduced by Howard [5], improved oscillation criteria for both (1) and the corresponding linear system

$$
Y^{\prime}=K(x) Z, \quad Z^{\prime}=G(x) Y
$$

are presented. Finally, using the techniques suggested by $F$. V. Atkinson [1, Chapter 10], estimates of oscillation which extend the estimates in [3] and which correspond to the estimates in [4], are determined.

The standard notations of matrix algebra and calculus are used throughout. In particular, if $A$ is an $n \times n$ matrix, then the symbols $A^{*}, A^{-1},|A|$ and $\operatorname{tr} A$ shall denote the transpose, inverse, determinant and trace of $A$ respectively. The symbol $I$ shall be used to denote the $n \times n$ identity matrix while 0 shall represent the zero matrix regardless of dimension.

1. Definitions and preliminary remarks. It is readily verified that if $\{Y(x), Z(x)\}$ is any solution of (1), then

$$
Y^{*} Z-Z^{*} Y \equiv C,
$$

$C$ an $n \times n$ constant matrix. For the linear system (2), the case $C=0$ is of special interest in the calculus of variations and, as a result, we define:

Definition 1. A solution pair $\{Y(x), Z(x)\}$ of (1) is said to be conjoined provided $Y^{*}(x) Z(x) \equiv Z^{*}(x) Y(x)$ on $[a, \infty)$.

We remark that the term "prepared" has also been used to describe such solution pairs.

Definition 2. A solution pair $\{Y(x), Z(x)\}$ of (1) is nontrivial provided

$$
Y^{*}(x) Y(x)+Z^{*}(z) Z(x)>0,
$$

i.e., is positive definite, on $[a, \infty)$.

The proof of the following lemma is readily established (e.g., see [2]).

LemMa 1. A solution pair $\{Y(x), Z(x)\}$ of $(1)$ is conjoined and nontrivial if and only if there exists a number $b, b \geqq a$, such that

(i) $Y^{*}(b) Z(b)=Z^{*}(b) Y(b)$,

(ii) $Y^{*}(b) Y(b)+Z^{*}(b) Z(b)>0$.

Definition 3. Let $\Gamma$ denote the collection to which the pair $\{Y(x), Z(x)\}$ belongs if and only if each of $Y$ and $Z$ is differentiable on $[a, \infty), Y^{*} Z \equiv Z^{*} Y$ and $Y^{*} Y+Z^{*} Z>0$. 
As mentioned previously, the oscillation results established in [2], [3] and in [6] were obtained under the condition that each of $K(x ; Y ; Z)$ and $G(x ; Y ; Z)$ is positive definite on $[a, \infty)$ for all differentiable pairs $\{Y, Z\}$. We now relax this condition by deleting the requirement that $G(x ; Y ; Z)$ be positive definite and by assuming, in the work which follows, only that $K(x ; Y ; Z)$ is positive definite on $[a, \infty)$ for all pairs $\{Y, Z\} \in \Gamma$.

Concerning the nontrivial, conjoined solution pairs $\{Y, Z\}$ of (1), the following theorem is an immediate extension of the results contained in [1, Chapter 10, §§2-5] and [4, Theorem 1.3].

THEOREM 1. If $\{Y(x), Z(x)\}$ is a nontrivial conjoined solution of (1), then the matrix $E(x)$ defined by

$$
E(x)=(Z+i Y)(Z-i Y)^{-1}, \quad i^{2}=-1,
$$

exists on $[a, \infty)$ and has the following properties:

(i) $E(x)$ is unitary and satisfies the differential equation

$$
E^{\prime}=2 i E H(x)
$$

where $H(x)$ is the Hermitian matrix

$$
H(x)=\left(Z^{*}+i Y^{*}\right)^{-1}\left(Z^{*} K^{\prime} Z+Y^{*} G Y\right)(Z-i Y)^{-1} .
$$

(ii) The characteristic roots $e_{j}(x), j=1,2, \cdots, n$, of $E(x)$ have the property that for each fixed $x, e_{j}(x)=+1$ for at least one $j$ if and only if $|Y(x)|=0$.

(iii) The functions $e_{j}(x), j=1,2, \cdots, n$, move monotonically and positively on the unit circle when they are at the point +1 .

(iv) Let $w_{j}(x)=\arg e_{j}(x), j=1,2, \cdots, n$, and assume that, for each $j, 0 \leqq w_{j}(a)<2 \pi$ and that each $w_{j}(x)$ is continued as a continuous function on $[a, \infty)$. Then

$$
|E(x)|=\exp \left\{i \sum_{j=1}^{n} w_{j}(x)\right\}
$$

and

$$
2 \int_{a}^{x} \operatorname{tr} H(t) d t=\sum_{j=1}^{n}\left[w_{j}(x)-w_{j}(a)\right] .
$$

(v) $|Y(x)|$ has at most a finite number of zeros on each compact subinterval of $[a, \infty)$ and the multiplicity of a zero of $|Y(x)|$, i.e. the dimension of the null space of $Y(x)$, is the same as the number of characteristic roots $e_{j}(x)$ of $E(x)$ having the value +1 . 
Definition 4. The differential system (1) is oscillatory provided each nontrivial conjoined solution pair $\{Y(x), Z(x)\}$ has the property that $|Y(x)|$ has infinitely many zeros on $[a, \infty)$.

2. A sufficient condition for oscillation. We consider first the special case of (1)

$$
Y^{\prime}=Z, \quad Z^{\prime}=G(z ; Y ; Z) Y .
$$

and establish the following theorem which improves the result in [5, Theorem 1].

Theorem 2. Let $Q(x)=\int_{a}^{x} G(t ; Y(t) ; Z(t)) d t$ and let $q(x)=\operatorname{tr} Q(x)$ $=\int_{a}^{x} \operatorname{tr} G(t ; Y(t) ; Z(t)) d t$. If $\lim _{x \rightarrow \infty} q(x)=\infty$ for all pairs $\{Y, Z\} \in \Gamma$, then (8) is oscillatory.

Proof. We employ an extension of the method presented in $[\mathbf{5}$, Theorem 1].

Suppose that the theorem is false. Then there exists a nontrivial conjoined solution pair $\{Y(x), Z(x)\}$ of (8) such that $|Y(x)|$ has at most a finite number of zercs on $[a, \infty)$. Let $b, b \geqq a$, have the property that $|Y(x)| \neq 0$ on $[b, \infty)$ and define the matrix $S(x)$ by the equation $S(x)=-Z(x) Y^{-1}(x)$ on $[b, \infty)$. Now $S$ is symmetric and $S^{\prime}$ $=G(x ; Y ; Z)+S^{2}$. In addition, the matrix $T(x)$ given by $T=S-Q$ is symmetric and $T^{\prime}=S^{2}=[T+Q]^{2}$.

Consequently $T^{\prime}$ is nonnegative definite and $T$ is "nondecreasing" in the sense that each of the characteristic roots of $T$ is a nondecreasing function on $[b, \infty)$.

Making use of the fact that $q(x) \rightarrow \infty$ as $x \rightarrow \infty$ together with the nondecreasing nature of $T(x)$, we have $\operatorname{tr}[T+Q] \rightarrow \infty$ as $x \rightarrow \infty$. In addition, it is clear that if $\operatorname{tr} A(x) \rightarrow \infty$ as $x \rightarrow \infty, A(x)$ a symmetric matrix, then $\lambda_{1}[A] \rightarrow \infty$ as $x \rightarrow \infty$, where $\lambda_{1}[A]$ denotes the maximum characteristic root of $A$. Thus we can conclude that there exists a point $c, c \geqq b$, such that $\operatorname{tr}[T(x)+Q(x)] \geqq \operatorname{tr}[T(c)+Q(x)] \geqq n+1$ and $\lambda_{1}[T(x)+Q(x)] \geqq 1+1 / n$ on $[c, \infty)$. It now follows that

$$
\operatorname{tr} T^{\prime}=\operatorname{tr}(T+Q)^{2} \geqq\left(1+g_{1} / n\right)^{2}, \quad g_{1} \equiv 1,
$$

and proceeding in a manner similar to the proof in $[5$, Theorem 1$]$

$$
\operatorname{tr} T(x) \geqq \operatorname{tr} T(c)+\int_{0}^{x}\left(1+g_{1} / n\right)^{2} .
$$

Thus

$$
\operatorname{tr}[T(x)+Q(x)] \geqq \operatorname{tr} T(c)+\operatorname{tr} Q(x)+\int_{c}^{x}\left(1+g_{1} / n\right)^{2}
$$


and since $\operatorname{tr}[T(x)+Q(x)] \geqq \operatorname{tr}[T(c)+Q(x)] \geqq n+1$, we have

$$
\operatorname{tr}[T(x)+Q(x)] \geqq n+1+\int_{c}^{x}\left(1+g_{1} / n\right) \equiv n+g_{2}(x) .
$$

Clearly

$$
\lambda_{1}[T(x)+Q(x)] \geqq 1+g_{2}(x) / n
$$

and, therefore,

$$
\operatorname{tr} T^{\prime}=\operatorname{tr}[T(x)+Q(x)]^{2} \geqq\left[1+g_{2} / n\right]^{2} .
$$

Now, as above,

$$
\operatorname{tr}[T(x)+Q(x)] \geqq n+1+\int_{a}^{x}\left[1+g_{2} / n\right]^{2}=n+g_{3}(x)
$$

and, inductively,

$$
\operatorname{tr}[T(x)+Q(x)] \geqq n+g_{k}(x)
$$

where

$$
g_{k}(x)=1+\int_{a}^{x}\left[1+g_{k-1} / n\right]^{2} .
$$

Note that the sequence $\left\{g_{k}(x)\right\}$ is pointwise increasing on $(c, \infty)$ since

$$
g_{k+1}(x)-g_{k}(x)=\int_{c}^{x}\left(\left[1+g_{k} / n\right]^{2}-\left[1+g_{k-1} / n\right]^{2}\right)
$$

and

$$
g_{2}(x)=1+(1+1 / n)^{2}(x-c)>g_{1}(x) \equiv 1 \text { on }(c, \infty) .
$$

Suppose that the functions $g_{k}(x)$ are bounded on an interval $[c, d]$, $d>c+n^{2} /(n+1)$ then $\lim _{k \rightarrow \infty} g_{k}(x)=f(x)$ exists and $f(x)$ satisfies

$$
f(x)=1+\int_{0}^{x}\left[1+\frac{f(t)}{n}\right]^{2} d t, f(c)=1
$$

But the only $C^{\prime}$ solution of this integral equation is

$$
f(x)=\frac{n^{2}(n+1)}{n^{2}+(n+1)(c-x)}-n
$$

which is unbounded as $x \rightarrow c+n^{2} /(n+1)$. Since $\operatorname{tr}[T(x)+Q(x)] \geqq f(x)$ if follows that $\operatorname{tr}[T+Q]$ is unbounded on any compact interval $[c, d]$, 
$d>c+n^{2} /(n+1)$ contradicting the fact that $\operatorname{tr}[T+Q]$ is continuous on $[b, \infty)$. We now conclude that each nontrivial, conjoined solution $\{Y, Z\}$ of $(8)$ has the property that $|Y|$ has infinitely many zeros on $[a, \infty)$, i.e., that $(8)$ is oscillatory.

As an immediate consequence of the theorem, we have the following improvement of Howard's result [5, Theorem 1]. of

COROLlaRy. If $\{Y(x), Z(x)\}$ is a nontrivial conjoined solution pair

$$
Y^{\prime}=Z, \quad Z^{\prime}=-G(x) Y,
$$

$G n \times n$ continuous and symmetric on $[a, \infty)$ and if $\int_{a}^{\infty} \operatorname{tr} G(x) d x=\infty$, then $|Y(x)|$ has infinitely many zeros on $[a, \infty)$, i.e., (10) is oscillatory.

The following two theorems are obtained exactly as in $[5$, Theorems 2 and 3] using the improved proof presented in Theorem 1. that

THEOREM 3. If there exists a positive differentiable function $g(x)$ such

$$
\lim _{x \rightarrow \infty} \int_{c}^{x}[1 / g(t)] d t=\infty
$$

and if

$$
J(x)=\int_{a}^{x}\left\{g(t) G(t ; Y ; Z)-\left[g^{\prime 2}(t) / 4 g(t)\right]\right\} I d t+\frac{g^{\prime}(x)}{2} I
$$

has the property that $\lim _{x \rightarrow \infty} \operatorname{tr} J(x)=\infty$ for all pairs $\{Y(x), Z(x)\} \in \Gamma$, then (8) is oscillatory.

Proof. Proceeding as in Theorem 2, assume there exists a nontrivial, conjoined solution $\{Y, Z\}$ of (8) such that $|Y(x)| \neq 0$ on $[b, \infty)$ for some $b \geqq a$.

Defining $S(x)$ by

$$
(1 / g(x)) \cdot S(x)=-Z(x) \cdot Y^{-1}(x), \quad x \geqq b,
$$

we obtain

$$
S^{\prime}=(1 / g)\left[S+\left(g^{\prime} / 2\right) I\right]^{2}+g G-\left(g^{\prime 2} / 4 g\right) I .
$$

Let $T(x)$ be defined by

$$
S(x)=T(x)+\int_{b}^{x}\left\{g(t) G(t ; Y ; Z)-\left[g^{\prime 2}(t) / 4 g(t)\right] I\right\} d t .
$$

Then $T^{\prime}=(1 / g)[T+J]^{2}$ and $T$ is symmetric with $T^{\prime}$ nonnegative definite. 
Paralleling the proof of Theorem 2, we have, in place of (9),

$$
f(x)=1+\int_{c}^{x}[1 / g(t)][1+f(t) / n]^{2} d t, \quad f(c)=1,
$$

with solution

$$
f(x)=\left\{n^{2} /\left[\frac{n^{2}}{n+1}-\int \frac{d t}{g(t)}\right]\right\}-n .
$$

A contradiction is obtained as in Theorem 2.

COROLlaRY. If there exists a positive differentiable function $g(x)$ such that $\int_{a}^{\infty}[1 / g(t)] d t=\infty$ and if

$$
J(x)=\int_{a}^{x}\left\{g(t) G(t)-\left[g^{\prime 2}(t) / 4 g(t)\right] I\right\} d t+\frac{g^{\prime}(x)}{2} I
$$

has the property that $\int_{a}^{\infty} \operatorname{tr} J(x) d x=\infty$, then (10) is oscillatory.

Turning now to the system (1), we have the following theorem. The proof will be omitted since it can be established exactly as in [5, Theorem 3] together with the improved proof of Theorems 2 and 3.

TheOREM 4. If there exists a positive differentiable function $g(x)$ such that the matrix

$$
\begin{aligned}
P(x)= & \int_{a}^{x}\left\{g(t) G(t ; Y ; Z)-K(t ; Y ; Z) \cdot g^{\prime 2}(t) / 4 g(t)\right\} d t \\
& +g^{\prime}(x) K(x ; Y ; Z)
\end{aligned}
$$

has the property

$$
\lim _{x \rightarrow \infty} \operatorname{tr} \int_{a}^{x} P(t) d t=\infty
$$

for all pairs $\{Y, Z\} \in \Gamma$, and if $[g(x) K(x ; Y ; Z)]^{-1} \geqq k(x) I$ for all such pairs $\{Y, Z\}$, where

$$
\lim _{x \rightarrow \infty} \int_{a}^{x} k(t) d t=\infty
$$

then (1) is oscillatory.

COROLLARY. If there exists a positive differentiable function $g(x)$ such that the matrix 


$$
M(x)=\int_{a}^{x}\left\{g(t) G(t)-K(t) \cdot g^{\prime 2}(t) / 4 g(t)\right\} d t+\frac{1}{2} g^{\prime}(x) K(x)
$$

has the property $\int_{a}^{\infty} \operatorname{tr} M(x) d x=\infty$, and if $[g(x) K(x)]^{-1} \geqq m(x) I$ where $\int_{a}^{\infty} m(x) d x=\infty$, then (2) is oscillatory.

We remark, in conclusion, that these results also extend the oscillation theorem for nonlinear matrix equations presented in $\$ 6$ of [5].

3. Estimates of oscillation. We now make use of Theorem 1 to extend the extimates presented in [3, Theorem 4.3].

Define the functions $u(x)$ and $v(x)$ on $[a, \infty)$ by

$$
\begin{aligned}
u(x)= & \underset{\{Y, Z\} \in \Gamma}{\text { g.l.b. }} \operatorname{tr}\{K(x ; Y ; Z)+G(x ; Y ; Z) \\
& \left.-\sqrt{ }\left([K(x ; Y ; Z)-G(x ; Y ; Z)]^{2}\right)\right\}, \\
v(x)= & \underset{\{Y, Z\} \in \Gamma . \text { l.u. }}{ } \operatorname{tr}\{K(x ; Y ; Z)+G(x ; Y ; Z) \\
& \left.+\sqrt{ }\left([K(x ; Y ; Z)-G(x ; Y ; Z)]^{2}\right)\right\},
\end{aligned}
$$

where $\sqrt{ }\left([K-G]^{2}\right)$ denotes the nonnegative definite square root of $(K-G)^{2}$.

Then, for any pair $\{Y, Z\} \in \Gamma$, it is readily verified that $u(x)$ $\leqq 2 \operatorname{tr} H(x) \leqq v(x)$, where $H(x)$ is given by (5).

As a consequence of this pair of inequalities and Theorem 1, we have

THEOREM 5. Let $Y(x), Z(x)$ be any nontrivial conjoined solution pair of (1) and $b>a$. Let $k$ be the greatest integer such that

$$
2 k n \pi<\int_{a}^{b} u(x) d x
$$

and $m$ the least nonnegative integer such that

$$
\int_{a}^{b} v(x) d x<2 m \pi \text {. }
$$

Finally, let $r=k$ if $k \geqq 0$ and $r=0$ if $k<0$. Then, the number of zeros of $|Y(x)|$ on $[a, b]$, multiple zeros being counted according to their multiplicity, is not less than $r$ and not greater than $n+m-1$. Moreover, if

$$
\lim _{x \rightarrow \infty} \int_{a}^{x} u(t) d t=\infty,
$$

then (1) is oscillatory; if 


$$
\lim _{x \rightarrow \infty} \int_{a}^{x} v(t) d t<\infty,
$$

then (1) is nonoscillatory, i.e., for every nontrivial conjoined solution pair $\{Y(x), Z(x)\}$ of (1), $|Y(x)|$ has at most a finite number of zeros on $[a, \infty)$.

\section{REFERENCES}

1. F. V. Atkinson, Discrete and continuous boundary problems, Math. in Science and Engineering, vol. 8, Academic Press, New York, 1964. MR 31 \#416.

2. G. J. Etgen, Oscillatory properties of certain nonlinear matrix differential systems of second order, Trans. Amer. Math. Soc. 122 (1966), 289-310. MR 32 \#7834.

3. - On the determinants of solutions of second-order matrix differential systems, J. Math. Anal. Appl. 18 (1967), 585-598. MR 35 \#4505.

4. - On the oscillation of solutions of second order self-adjoint matrix differential equations, J. Differential Equations 6 (1969), 187-195. MR 39 \#3091.

5. H. C. Howard, Oscillation criteria for matrix differential equations, Canad. J. Math. 19 (1967), 184-199. MR 35 \#3126.

6. E. C. Tomastik, Oscillation of nonlinear matrix differential equations of second order, Proc. Amer. Math. Soc. 19 (1968), 1427-1431. MR 38 \#372.

University of Houston, Houston, TeXas 77004 